\title{
Der mittlere Druck der Flüssigkeit
}

\author{
Tain-Yen Hsia, MD, MSc
}

\footnotetext{
From the Department of Cardiothoracic Surgery, Great Ormond Street Hospital for Children; and the Institute of Cardiovascular Sciences, University College London, London, United Kingdom.

Disclosures: T-Y.H. is the principle investigator of a Transatlantic Network of Excellence Grant from Fondation Leducq, Paris, France.

Received for publication Dec 14, 2016; accepted for publication Dec 20, 2016; available ahead of print Jan 31, 2017.

Address for reprints: Tain-Yen Hsia, MD, MSc, UCL, Cardiac Unit, 7th Floor Nurses Home, Great Ormond St Hospital for Children, NHS Foundation Trust, London, United Kingdom WC1N 3JH (E-mail: hsiat@gosh. nhs.uk).

J Thorac Cardiovasc Surg 2017;153:921-2

$0022-5223 / \$ 36.00$

Copyright (C) 2017 by The American Association for Thoracic Surgery

http://dx.doi.org/10.1016/j.jtcvs.2016.12.024
}

E. H. Weber is regarded as one of the fathers of experimental psychology and physiology (Figure 1). He and his two younger brothers are considered the Da Vincis of their time because of their seminal contributions to wide-ranging areas in medicine and physiology. Among their numerous discoveries were those in the 1825 publication of Wellenlehre (wave teachings), in which they formulated the basic laws of hydrodynamics and were the first to apply this branch of physics to the circulation of the blood. In 1850, realizing the importance of blood volume and vascular capacitance in a closed circuit, Weber described the equilibration of pressures throughout the circulatory system at zero flow (ie, cardiac arrest) as "mittlere Druck der Flüssigkeit" (mean pressure of the liquid). ${ }^{1}$ Nearly 50 years later, on February 24, 1897, E. H. Starling, in his Arris and Gale Lecture at the Royal College of Surgeons of England, called to attention that this mean circulatory filling pressure (Pmcf) describes the fullness of the circulatory system and is inherently a measure of venous capacitance or venomotor activity.

In the Fontan circulation, the paradoxic association of systemic venous hypertension with pulmonary arterial hypotension results in reduced cardiac preload and loss of venous capacitance. Because Pmcf provides an estimate of the upstream pressure that determines the rate of flow returning to the heart, its quantification, along with central venous pressure (CVP, or Fontan pressure), could be invaluable as a physiological gauge of venous well-being in patients with Fontan circulations. The problem, however, resides in how to measure Pmcf without stopping a patient's heart and waiting for blood to redistribute so that all the vascular pressures are equalized. Similarly, a less-invasive method to assess CVP in the ambulatory setting would certainly be a welcome tool for those looking after patients with Fontan circulations.

In this issue of the Journal, Masutani and colleagues ${ }^{3}$ present their study of a cohort of patients with Fontan

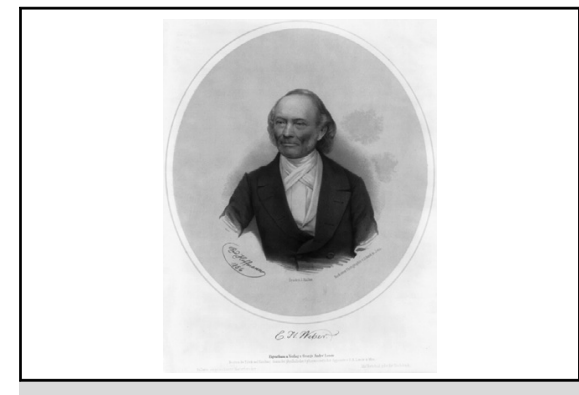

Ernst Heinrich Weber (lithograph by Rudolf Hoffmann 1856).

Central Message

In patients with Fontan circulations, central venous and mean circulatory filling pressures can be estimated peripherally to provide valuable insights into their venous circulatory well-being.

See Article page 912.

circulations to estimate CVP from peripheral venous pressure (PVP) and Pmcf from the arm equilibrium pressure (Parm-occ), which required acute and rapid occlusion of arterial and venous flow to the upper arm. Masutani and colleagues $^{3}$ derived a regression equation that could predict CVP from PVP and demonstrated close correlation between Parm-occ and Pmcf (which they estimated by applying a Valsalva maneuver during diagnostic catheterization). They concluded that the adaptation of PVP and Parm-occ could assist in the monitoring of patients with Fontan circulations and guide pharmacologic interventions to improve hemodynamics. Although both the study and methodology employed were elegant, the concept that PVP is a surrogate for CVP in Fontan patients is intuitive. PVP approximates CVP in patients with Fontan circulations because the difference between PVP and CVP is simply a reflection of the venous resistance from the periphery to the vena cava. With obligatory loss of venous capacitance and resistance, along with inability to lower CVP through atrial filling, the difference between PVP and CVP becomes small and predictable. Interestingly, the observation of the abolition of a pressure difference between PVP and CVP was first seen in another vascular region of interest in patients with Fontan circulations, the splanchnic circulation. In 2001, the Great Ormond Street group ${ }^{4}$ demonstrated that the portal venous and hepatic venous pressures (ie, CVP) were essentially equal in patients with Fontan circulations. Whereas a transhepatic venous pressure gradient of 2 to 


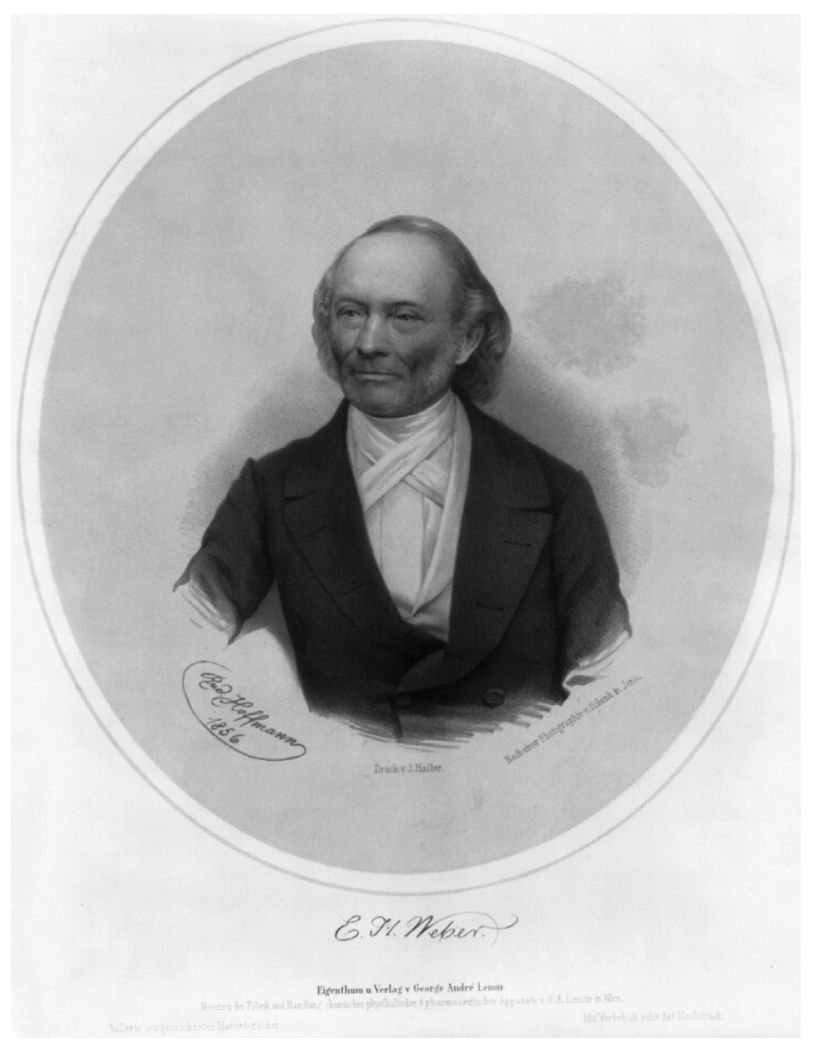

FIGURE 1. Ernst Heinrich Weber (lithograph by Rudolf Hoffmann, 1856).

$3 \mathrm{~mm} \mathrm{Hg}$ existed in healthy subjects, its absence in patients with Fontan circulations signified a loss of resistance and capacitance in the hepatic sinusoids. This finding further supported the notion that the splanchnic venous circulation is a privileged domain in patients with Fontan circulations that is particularly susceptible to stress and disturbances, such as gravity and a paralyzed diaphragm.

Because most of us are unfamiliar with the concept of Pmcf, its clinical application in the Fontan circulation is less intuitive. Nonetheless, its importance as a measure of vascular capacitance (fullness of the circulatory system) and its role in normal and pathologic circulations (such as congestive heart failure) have been well recognized by classic physiologists such as Starling, Guyton, and Rothe. ${ }^{5}$
Masutani and colleagues ${ }^{3}$ suggest that in patients with Fontan circulations, increased Pmcf is a compensatory mechanism to maintain preload, and its modulation by nitrates and angiotensin-converting enzyme inhibitors may provide a pharmacologic avenue to lower Fontan pressures. Therefore, as pointed out by Masutani and colleagues, ${ }^{3}$ a less invasive method to assess Pmcf not only adds to our knowledge of the Fontan circulation but also introduces a new clinical marker that can provide insights into the selection and assessment of individualized therapies. The validity of the proposed Fontan pressure regression equation, however, and that of Pmcf (Parm-occ) as a prognostic predictor of Fontan failure or attrition, will require a well-designed, prospective study of a much larger cohort of patients.

Incidentally, another of E. H. Weber's great contributions was his startling discovery that the heart action can be slowed and even stopped by vagal stimulation. This was the first time that inhibition, as opposed to excitation, had been described in nerve action. Weber was also the first person to study the effects of the Valsalva maneuver when he demonstrated in himself the provocation of bradycardiaand possibly cardiac arrest-at a scientific meeting in 1850 in Leipzig, where he lost consciousness and had a convulsion. ${ }^{6}$ One must wonder whether he was simply trying to prove the existence of his beloved "mittlere Druck der Flüssigkeit." If only he had known that all he needed was a tourniquet and a peripheral intravenous tube.

\section{References}

1. Weber EH. Über die Anwendung der Wellenlehre auf die Lehre vom Kreislauf des Blutes und insbesondere auf die Pulslehre. In: Berichte über die Verhandlungen der Königlich Sächsischen Gesellschaft der Wissenschaften zu Leipzig [Reports on the proceedings of the Royal Saxon Society of Sciences in Leipzig]. Leipzig: Weidmannsche Buchhandlung; 1850.

2. Starling EH. The effects of heart failure on the circulation. Lancet. 1897;1:652-5.

3. Masutani S, Kurishima C, Yana A, Kuwata S, Iwamoto Y, Saiki H, et al. Assessment of central venous physiology of Fontan circulation using peripheral venous pressure. J Thorac Cardiovasc Surg. 2017;153:912-20.

4. Hsia TY, Khambadkone S, Deanfield JE, Taylor JF, Migliavacca F, De Leval MR. Subdiaphragmatic venous hemodynamics in the Fontan circulation. J Thorac Cardiovasc Surg. 2001;121:436-47.

5. Rothe CF. Mean circulatory filling pressure: its meaning and measurement. J Appl Physiol (1985). 1993;74:499-509.

6. Jellinek EH. The Valsalva manoeuvre and Antonio Valsalva (1666-1723). J R Soc Med. 2006;99:448-51. 